\title{
NUMERICAL STUDIES ON 2-DIMENSIONAL REACTION-DIFFUSION EQUATIONS
}

\author{
S. TANG ${ }^{\prime}$, S. QIN' \\ and R. O. WEBER ${ }^{2}$
}

(Received 7 August 1992; revised 28 April 1992)

\begin{abstract}
Various initial and boundary value problems for a 2-dimensional reaction-diffusion equation are studied numerically by an explicit Finite Difference Method (FDM), a Galerkin and a Petrov-Galerkin Finite Element Method (FEM). The results not only show the transition processes from different local initial disturbances to quasitravelling waves, but also demonstrate the long term behaviour of the solutions, which is determined by the system itself and does not depend on the details of the initial disturbances.
\end{abstract}

\section{Introduction}

Many complicated natural phenomena, such as the spreading of bushfires and epidemics, and the nonlinear evolution of a population in a 2-dimensional habitat, (in which the balance of reaction and diffusion are concerned) can be modelled by a 2 -dimensional reaction-diffusion equation

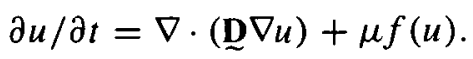

Here $u$ is a dimensionless temperature or population, $\partial u / \partial t$ is the rate of increase of $u$ with time $t, \nabla$ is the gradient operator in 2-dimensional space, $\underset{d}{\mathbf{D}}$ is a constant second order tensor measuring the diffusivity of the media, and $f(u)$ is a nonlinear function of $u$ representing the effect of reaction or multiplication. We place a constant coefficient $\mu$ before $f(u)$ for convenience.

'Dept of Mechanics, Peking University, Beijing 100871, China

${ }^{2}$ Dept of Mathematics, University of NSW, ADFA, Canberra ACT 2600, Australia

(C) Australian Mathematical Society, 1993, Serial-fee code 0334-2700/93 
Assuming $\lambda_{1}$ and $\lambda_{2}$ are the principal values of $\underset{\sim}{\mathbf{D}}$ and $x$ and $y$ are coordinates along the principle axes, (1) can be written as

$$
\frac{\partial u}{\partial t}=\lambda_{1} \frac{\partial^{2} u}{\partial x^{2}}+\lambda_{2} \frac{\partial^{2} u}{\partial y^{2}}+\mu f(u) .
$$

If we rescale $t, x$ and $y$ by

$$
T=\mu t, \quad X=\sqrt{\frac{\mu}{\lambda_{1}}} x, \quad Y=\sqrt{\frac{\mu}{\lambda_{2}}} y,
$$

(2) becomes

$$
\frac{\partial u}{\partial T}=\frac{\partial^{2} u}{\partial X^{2}}+\frac{\partial^{2} u}{\partial Y^{2}}+f(u)
$$

Introducing polar coordinates in the $(X, Y)$ plane, $(4)$ can be written as

$$
\frac{\partial u}{\partial T}=\frac{\partial^{2} u}{\partial r^{2}}+\frac{1}{r} \frac{\partial u}{\partial r}+\frac{1}{r^{2}} \frac{\partial^{2} u}{\partial \theta^{2}}+f(u) .
$$

From (5) we see that, when $r \gg r_{0}$, where $r_{0}$ is the space scale of initial disturbances, the second and third terms on the right hand side of (5) become very small and can be neglected. This means that the large scale response to a local disturbance can be approximately described by a one-dimensional reaction-diffusion equation, which possesses long term travelling wave solutions for several choices of $f(u)$, say $f(u)=u^{n}(1-u), n=1,2$. With $n=1$, the one-dimensional equation is often known as the Fisher-Kolmogorov equation. See for example, Aronson and Weinberger [2], Tang and Weber [5] and Tang, Qin and Weber [4].

Based on this asymptotic analysis, we expect that any local disturbance will grow fully and after a long period forms a quasi-travelling wave, which has a circular wave front with a velocity changing with distance $r$ due to the 2dimensional effect. Obviously, the circular wave front solution to (4) or (5) is an elliptic wave front solution to (2) according to the transformation (3). A theoretical analysis of this "hair trigger" effect is given by Aronson and Weinberger [2].

In this paper, several numerical schemes for solving the initial-boundary value problems of a 2-dimensional reaction-diffusion equation are presented. The numerical schemes from the Galerkin and Petrov-Galerkin FEM usually have excellent accuracy and stability $[4,5]$ and have been successfully used to solve the 2-dimensional nonlinear dispersive wave equations, but they require 
a long CPU time, so they are used to solve transition problems in a small space and short period. Generally speaking, the numerical schemes from FDM usually cause pseudo-oscillations, but our numerical experiments show that this is not the case for our problems, even when an explicit FDM is used. Since the explicit FDM need not invert a large matrix and do iterations, it is fast and is therefore suitable for long term studies in large spaces.

In Section 2, after presenting the mathematical models to be solved, their numerical models, especially the Galerkin FEM model, are shown in some detail. Various numerical results are shown in Section 3 and some conclusions are summarized in Section 4.

\section{Numerical schemes}

We begin by adding some initial and boundary value conditions to (2) to complete the mathematical models which we wish to consider.

As a fundamental model, we look for $u(x, y, t)$ satisfying

$$
\begin{aligned}
& \frac{\partial u}{\partial t}=\lambda_{1} \frac{\partial^{2} u}{\partial x^{2}}+\lambda_{2} \frac{\partial^{2} u}{\partial y^{2}}+\mu u(1-u), \quad t>0, \quad(x, y) \in \Omega, \\
& u(x, y, 0)=u^{0}(x, y), \quad 0 \leq u^{0} \leq 1, \quad(x, y) \in \Omega_{0} \subset \Omega, \\
& \text { n. } \nabla u=0, \quad t>0, \quad(x, y) \in \partial \Omega
\end{aligned}
$$

or

$$
u=0, \quad t>0, \quad(x, y) \in \partial \Omega .
$$

where $\Omega=\{(x, y):|x|<a,|y|<b\}, \partial \Omega=\{(x, y):|x|=a$ or $|y|=b\}, \mathbf{n}$ is a unit normal vector of boundary $\partial \Omega, \Omega_{0}$ is a local subset of $\Omega$ reflecting the fact that the initial disturbance $u^{0}(x, y)$ has compact support. If $\Omega$ is big enough compared with $\Omega_{0}$, and $t$ is not sufficiently large (so that the boundary is not yet conscious of the existence of the disturbance), then the boundary condition (6.4) is available. Otherwise, (6.3), the so called natural boundary condition, is in good agreement with practice.

In all the numerical schemes being shown below, $\Omega$ is divided into a regular, rectangular grid with node points $\left(x_{n}, y_{m}\right)$

$$
\begin{aligned}
x_{n} & =-a+(n-1) h, & n & =1,2, \ldots, N \\
y_{m} & =-b+(m-1) k, & m & =1,2, \ldots, M
\end{aligned}
$$

where $h=(2 a) /(N-1)$ and $k=(2 b) /(M-1)$ are the length and the width of the grid, see Figure 1. 


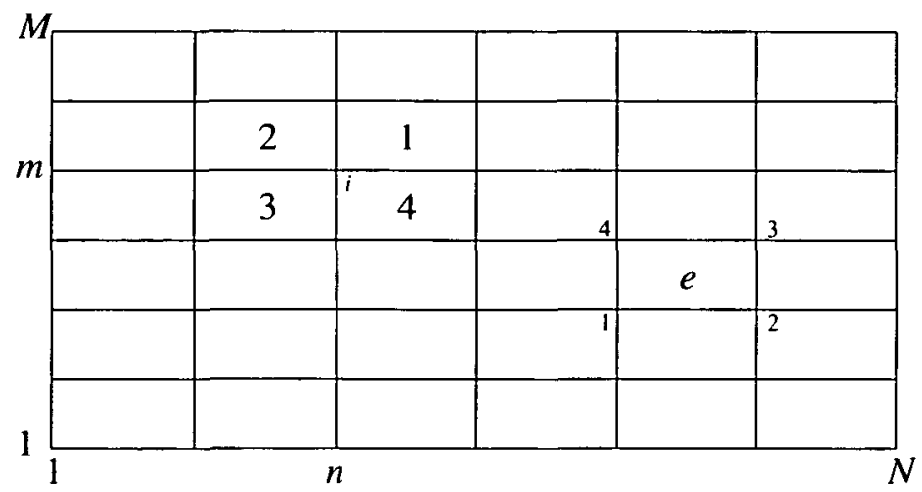

FIGURE 1. Division of grids, elements and nodes.

Writing $t_{p}=p \tau, p=1,2, \ldots$, where $\tau>0$ is the time step, we can then discretize $u(x, y, t)$ into

$$
u_{m m}^{p}=u\left(x_{n}, y_{m}, t_{p}\right) \text {. }
$$

2.1. FDM scheme The derivatives of $u$ at the inner node points are approximated by central finite differences which have an accuracy of second order. Then, using the Newtonian forward FDM to integrate with respect to $t$ for the inner node points, we have

$$
\begin{aligned}
& u_{n, m}^{p+1}=u_{n, m}^{p}+\tau\left\{\frac{\lambda_{1}}{h^{2}}\left[u_{n+1, m}^{p}-2 u_{n, m}^{p}+u_{n-1, m}^{p}\right]+\frac{\lambda_{2}}{k^{2}}\left[u_{n, m+1}^{p}-2 u_{n, m}^{p}+u_{n, m-1}^{p}\right]\right. \\
&+\left.\mu u_{n, m}^{p}\left(1-u_{n, m}^{p}\right)\right\} \\
& n=2, \ldots, N-1 \quad m=2, \ldots, M-1
\end{aligned}
$$

For the boundary condition (6.4), we should use

$$
\begin{cases}u_{1, m}^{p+1}=u_{N, m}^{p+1}=0, & m=1,2, \ldots, M \\ u_{n, 1}^{p+1}=u_{n, M}^{p+1}=0, & n=1,2, \ldots, N .\end{cases}
$$

For the boundary condition (6.3), from the approximation for any differentiable function $F(x)$

$$
F^{\prime}(x)=[4 F(x+h)-F(x+2 h)-3 F(x)] /(2 h)+\mathrm{O}\left(h^{2}\right)
$$


and similar approximations to calculate the normal gradients at boundary using the values near and on the boundary and put them to be zero, we obtain

$$
\begin{cases}u_{1, m}^{p+1}=\frac{1}{3}\left(4 u_{2, m}^{p+1}-u_{3, m}^{p+1}\right) & m=1,2, \ldots, M \\ u_{N, m}^{p+1}=\frac{1}{3}\left(4 u_{N-1, m}^{p+1}-u_{N-2, m}^{p+1}\right) & m=1,2, \ldots, M \\ u_{n, 1}^{p+1}=\frac{1}{3}\left(4 u_{n, 2}^{p+1}-u_{n, 3}^{p+1}\right) & n=1,2, \ldots, N \\ u_{n, M}^{p+1}=\frac{1}{3}\left(4 u_{n, M-1}^{p+1}-u_{n, M-2}^{p+1}\right) & n=1,2, \ldots, N .\end{cases}
$$

All corner points of $\Omega$, such as $u_{1,1}^{p+1}$, are calculated twice by this scheme, so we take the mean value for further computations and for our final result.

Starting from $u_{n, m}^{0}=u^{0}\left(x_{n}, y_{m}\right)$, we are able to get $u_{n, m}^{p}$ step by step for any integer $p>0$ using (7) and (8) or (7) and (9). It is obvious that this scheme has accuracy of $\mathrm{O}\left(\tau, h^{2}+k^{2}\right)$.

2.2. Galerkin FEM scheme for boundary condition (6.3) In this scheme, we take every rectangular grid as an element, so we have $E=(N-1)(M-1)$ elements and $I=N \times M$ node points, both of which are given global numbers $e$ and $i$ column by column ( $y$-direction). In this way, the 2 -dimensional array $u_{n, m}$ has been changed to a 1 -dimensional array $u_{i}$, with the relation

$$
i=(n-1) M+m .
$$

Around every node point $i$, there are four local elements 1 to 4 , counted by their locations in four quadrants. On the other hand, for every element $e$, there are four local node points 1 to 4, see Figure 1 .

Introducing the local dimensionless coordinates

$$
\zeta=2\left(x-x_{i}\right) / h, \quad \eta=2\left(y-y_{i}\right) / k,
$$

we define the sets of interpolation and weighting functions

$$
\Phi_{i}(\zeta, \eta)= \begin{cases}\phi_{\alpha}, & (\zeta, \eta) \in \text { element } \alpha, \alpha=1,2,3,4 \\ 0, & \text { otherwise }\end{cases}
$$

where

$$
\left\{\begin{array}{l}
\phi_{1}=(1-\zeta)(1-\eta) / 4 \\
\phi_{2}=(1+\zeta)(1-\eta) / 4 \\
\phi_{3}=(1+\zeta)(1+\eta) / 4 \\
\phi_{4}=(1-\zeta)(1+\eta) / 4
\end{array}\right.
$$




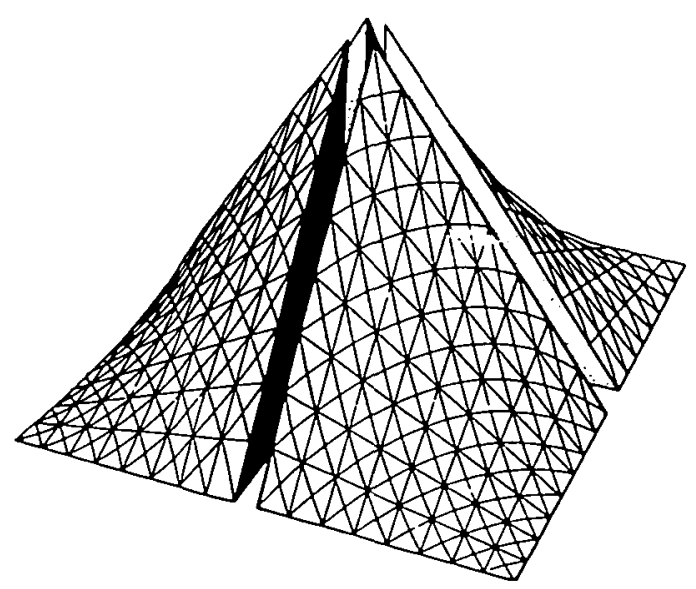

FIGURE 2. Function $\Phi_{i}(\zeta, \eta)$.

Obviously, $\Phi_{i}$ are piecewise continuous functions which are linear along the boundaries of the elements, and $\Phi_{i}(0,0)=1$, see Figure 2 .

Using the coordinate transformations (10), we transfer $\Phi_{i}(\zeta, \eta)$ into $\Phi_{i}(x, y)$, which are then used to approximate $u$ and $u^{2}$ by the expansions

$$
\begin{aligned}
u(x, y, t) & \doteq u_{j}(t) \Phi_{j}(x, y), \\
u^{2}(x, y, t) & \doteq u_{j}^{2}(t) \Phi_{j}(x, y) .
\end{aligned}
$$

Hereafter the Einstein summation convention for $j=1, \ldots, I$ is used. Generally speaking, the square of (13) is not identical to (14), however, it is equal at all node points.

Substituting (13) and (14) into (6.1) and taking the weighted residuals to be zero for every weighting function $\Phi_{i}$, after integrating by parts and considering the boundary condition (6.3), we have

$$
m_{i j} \dot{u}_{j}+s_{i j} u_{j}+\mu m_{i j} u_{j}^{2}=0,
$$

where $s_{i j}=n_{i j}-\mu m_{i j}$, and

$$
\begin{aligned}
& m_{i j}=\int_{\Omega} \Phi_{i} \Phi_{j} d \Omega=\sum_{e=1}^{E} \Delta_{i r}^{e} \Delta_{j s}^{e} m_{r s}^{e}, \\
& m_{r s}^{e}=\frac{h k}{4} \int_{-1}^{1} \int_{-1}^{1} \phi_{r} \phi_{s} d \zeta d \eta=\frac{h k}{36}\left(\begin{array}{llll}
4 & 2 & 1 & 2 \\
2 & 4 & 2 & 1 \\
1 & 2 & 4 & 2 \\
2 & 1 & 2 & 4
\end{array}\right),
\end{aligned}
$$




$$
\begin{aligned}
n_{i j} & =\int_{\Omega}\left(\lambda_{1} \frac{\partial \Phi_{i}}{\partial x} \frac{\partial \Phi_{j}}{\partial x}+\lambda_{2} \frac{\partial \Phi_{i}}{\partial y} \frac{\partial \Phi_{j}}{\partial y}\right) d x d y=\sum_{e=1}^{E} \Delta_{i r}^{e} \Delta_{j s}^{e} n_{r s}^{e}, \\
n_{r s}^{e} & =\frac{h k}{4} \int_{-1}^{1} \int_{-1}^{1}\left[\frac{4 \lambda_{1}}{h^{2}} \frac{\partial \phi_{r}}{\partial \zeta} \frac{\partial \phi_{s}}{\partial \zeta}+\frac{4 \lambda_{2}}{k^{2}} \frac{\partial \phi_{r}}{\partial \eta} \frac{\partial \phi_{s}}{\partial \eta}\right] d \zeta d \eta \\
& =\frac{1}{6 h k}\left[\begin{array}{cccc}
2\left(\lambda_{2} h^{2}+\lambda_{1} k^{2}\right) & \lambda_{2} h^{2}-2 \lambda_{1} k^{2} & -\left(\lambda_{2} h^{2}+\lambda_{1} k^{2}\right) & -2 \lambda_{2} h^{2}+\lambda_{1} k^{2} \\
\lambda_{2} h^{2}-2 \lambda_{1} k^{2} & 2\left(\lambda_{2} h^{2}+\lambda_{1} k^{2}\right) & -2 \lambda_{2} h^{2}+\lambda_{1} k^{2} & -\left(\lambda_{2} h^{2}+\lambda_{1} k^{2}\right) \\
-\left(\lambda_{2} h^{2}+\lambda_{1} k^{2}\right) & -2 \lambda_{2} h^{2}+\lambda_{1} k^{2} & 2\left(\lambda_{2} h^{2}+\lambda_{1} k^{2}\right) & \lambda_{2} h^{2}-2 \lambda_{1} k^{2} \\
-2 \lambda_{2} h^{2}+\lambda_{1} k^{2} & -\left(\lambda_{2} h^{2}+\lambda_{1} k^{2}\right) & \lambda_{2} h^{2}-2 \lambda_{1} k^{2} & 2\left(\lambda_{2} h^{2}+\lambda_{1} k^{2}\right)
\end{array}\right],
\end{aligned}
$$

where the Boolean matrix is defined as

$$
\Delta_{i s}^{e}= \begin{cases}1, & \text { if the local node } s \text { coincides with the global node } i, \\ 0, & \text { otherwise. }\end{cases}
$$

It is easy to see that every $u_{i}$ is related with the values of $u$ at the nine nodes of the four local elements surrounding point $i$, but these points are scattered in a section with $2(M+1)+1=2 M+3$ points in the list of global node points, so both $m_{i j}$ and $n_{i j}$ are banded matrices with a bandwidth $2 M+3$.

Integrating (15) with respect to $t$ by a Crank-Nicolson scheme, we get a system of nonlinear algebraic equations, which are linearized using Newton-Raphson iteration to be a system of linear algebraic equations

$$
A_{i j} u_{j}^{p+1, r+1}=B_{i}, \quad i=1,2, \ldots, I
$$

with

$$
\begin{aligned}
A_{i j} & =m_{i j}+\frac{\tau}{2} s_{i j}+\tau \mu m_{i j} u_{j}^{p+1 . r}, \\
B_{i} & =m_{i j}\left[u_{j}^{p}-\frac{\tau \mu}{2}\left(u_{j}^{p}\right)^{2}+\frac{\tau \mu}{2}\left(u_{j}^{p+1 . r}\right)^{2}\right]-\frac{\tau}{2} s_{i j} u_{j}^{p}
\end{aligned}
$$

and where $u_{j}^{p, r}$ is the value of $u_{j}$ at $t_{p}=p \tau$ and is the iteration at the $r$ th step.

Since $A_{i j}$ is a banded matrix, (16) is solved by calling a standard subroutine for a system of linear algebraic equations with a banded matrix. Knowing $u_{j}^{0}$, we solve (16) step by step until any time $t_{p}$. This scheme has higher accuracy both in space and time than the FDM.

2.3. Petrov-Galerkin FEM scheme for boundary condition (6.4) This scheme is similar to that used to solve the 2-dimensional nonlinear dispersive wave equation, the RKP equation, by Grimshaw and Tang [3], so we only describe this scheme briefly. 
First we rewrite (6.1) as

$$
\frac{\partial u}{\partial t}-\lambda_{1} \frac{\partial^{2} u}{\partial x^{2}}-\mu u(1-u)=\lambda_{2} \frac{\partial^{2} u}{\partial y^{2}} .
$$

To get the value of $u$ at $t_{p+1}$ we use its value at $t_{p}$ and the method of cubic spline interpolation to calculate $\partial^{2} u / \partial y^{2}$, which satisfies the boundary condition at $y= \pm b$ and is treated as a forcing term in (17). Then we integrate the resultant 1 -dimensional (in space) forced equation considering $y_{m}$ as a parameter with the boundary condition (6.3) at $x= \pm a$. In doing so, a Petrov-Galerkin FEM is used. For details, see Grimshaw and Tang [3], Tang and Weber [5].

This scheme also has higher accuracy in space than the FDM, but is only of first order accuracy in time.

\section{Numerical results}

In this section we show some examples chosen from our numerical results.

3.1. Initial disturbance with a flat top in a small space: FEM In this example, we set $\lambda_{1}=0.1, \lambda_{2}=0.01, \mu=0.1, a=2, b=1, h=k=0.05, \tau=0.01$ and

$$
u^{0}(x, y)= \begin{cases}1, & x^{2}+4 y^{2} \leq 0.25 \\ \exp \left[-10\left(x^{2}+4 y^{2}-0.25\right)\right], & \text { otherwise }\end{cases}
$$

Using the Galerkin FEM scheme, we get the results at $t=0,1,2,4$ shown by 3-dimensional contour plots of $u$ ( $z$-axis in the figures) in Figure 3(a)-(d).

From this example we see that, even though the initial disturbance has a flat top in the middle, where diffusion is zero, there is strong diffusion at the edge, and as time goes on, the top falls at first while the disturbance is spreading. Since $\lambda_{1}>\lambda_{2}$, the spread in the $x$-direction is faster than that in the $y$-direction.

3.2. Initial disturbance with a concave top in a small space: FEM In this example, the parameters $\lambda_{1}, \lambda_{2}, \mu, a, b$ and $\tau$ are taken to be the same as those in example 1 , but $h=k=0.1$ and

$$
u^{0}(x, y)= \begin{cases}1-\left[0.09-\left(x^{2}+4 y^{2}\right)\right]^{\frac{1}{2}}, & x^{2}+4 y^{2} \leq 0.09 \\ \exp \left[-10\left(x^{2}+4 y^{2}-0.09\right)\right], & \text { otherwise }\end{cases}
$$

Figure 4(a-d) shows the contour plots of $u$ at $t=0,0.4,1$ and 4 . 

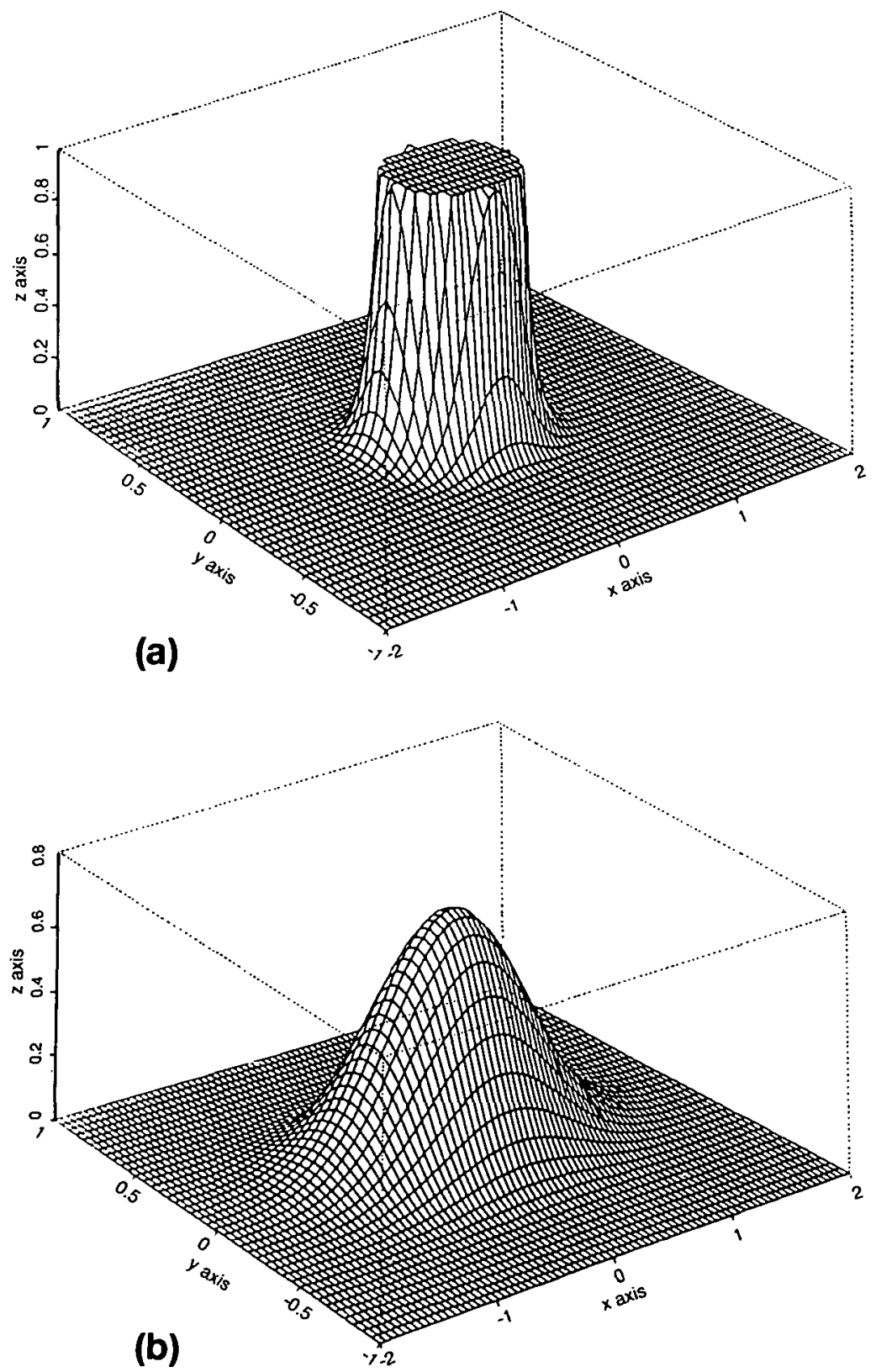

FIGURE 3. Contour plots for $u$ due to initial disturbance with a flat top in a small space. (a) $t=0$ and (b) $t=1$ (FEM) (for (c) and (d) see overleaf). 


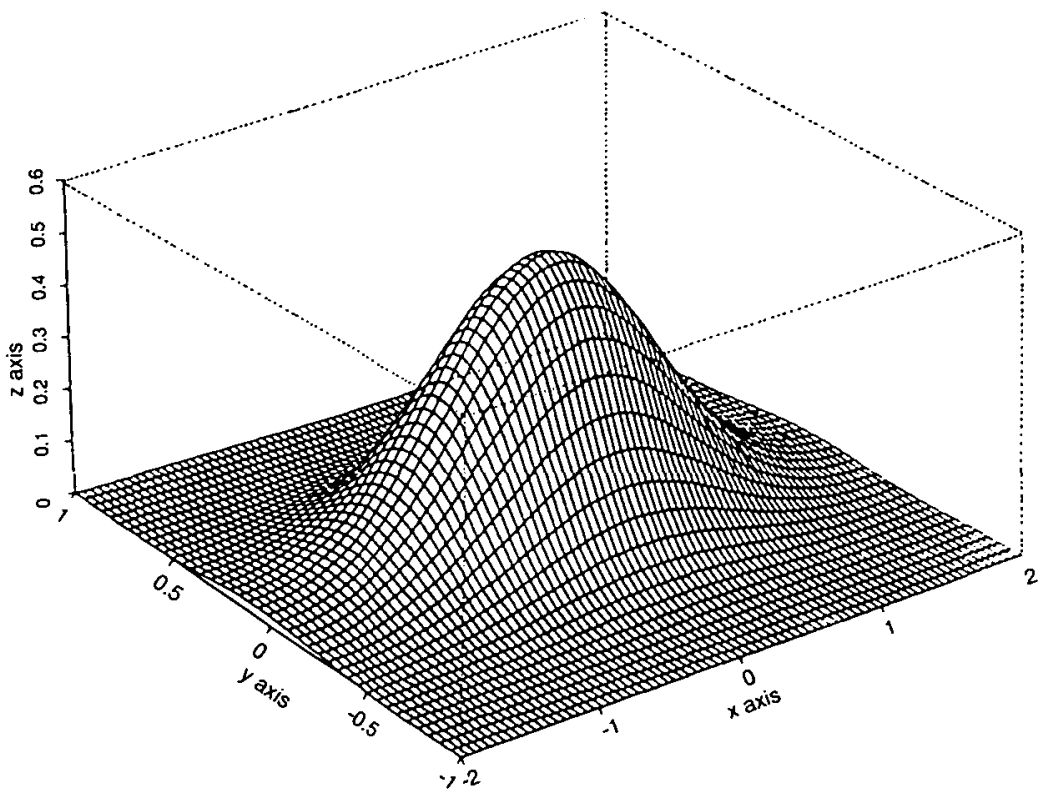

(c)

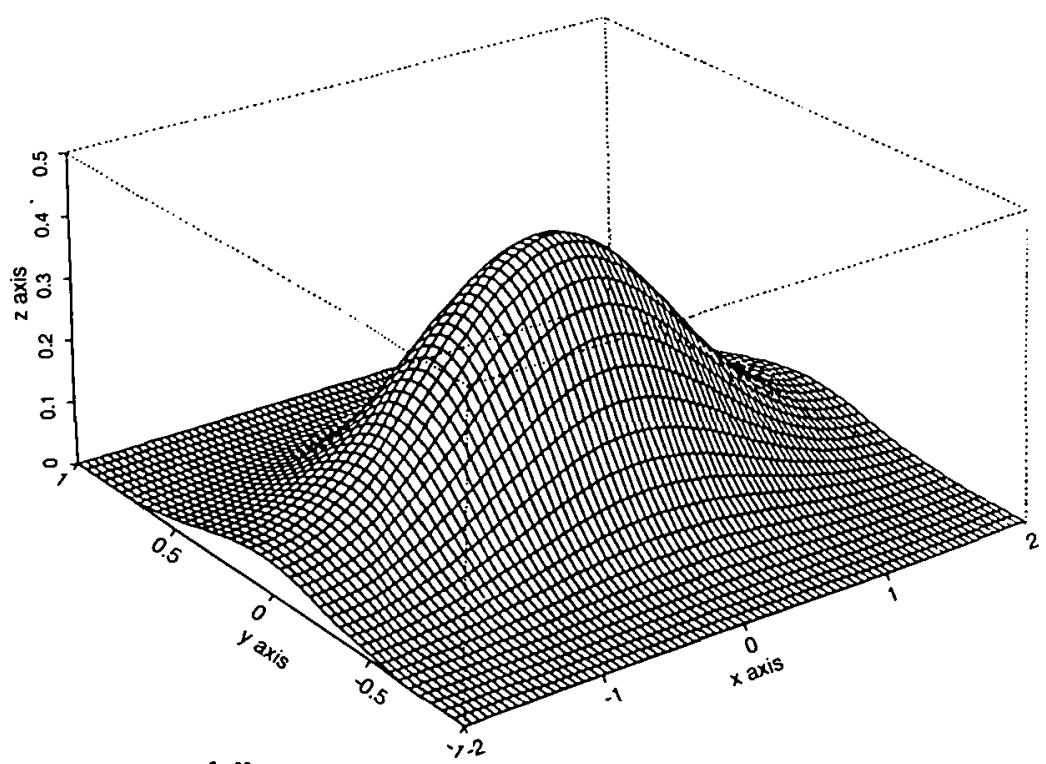

(d)

FIGURE 3. Contour plots for $u$ due to initial disturbance with a flat top in a small space. (c) $t=2$ and (d) $t=3$ (FEM) (for (a) and (b) see previous page). 

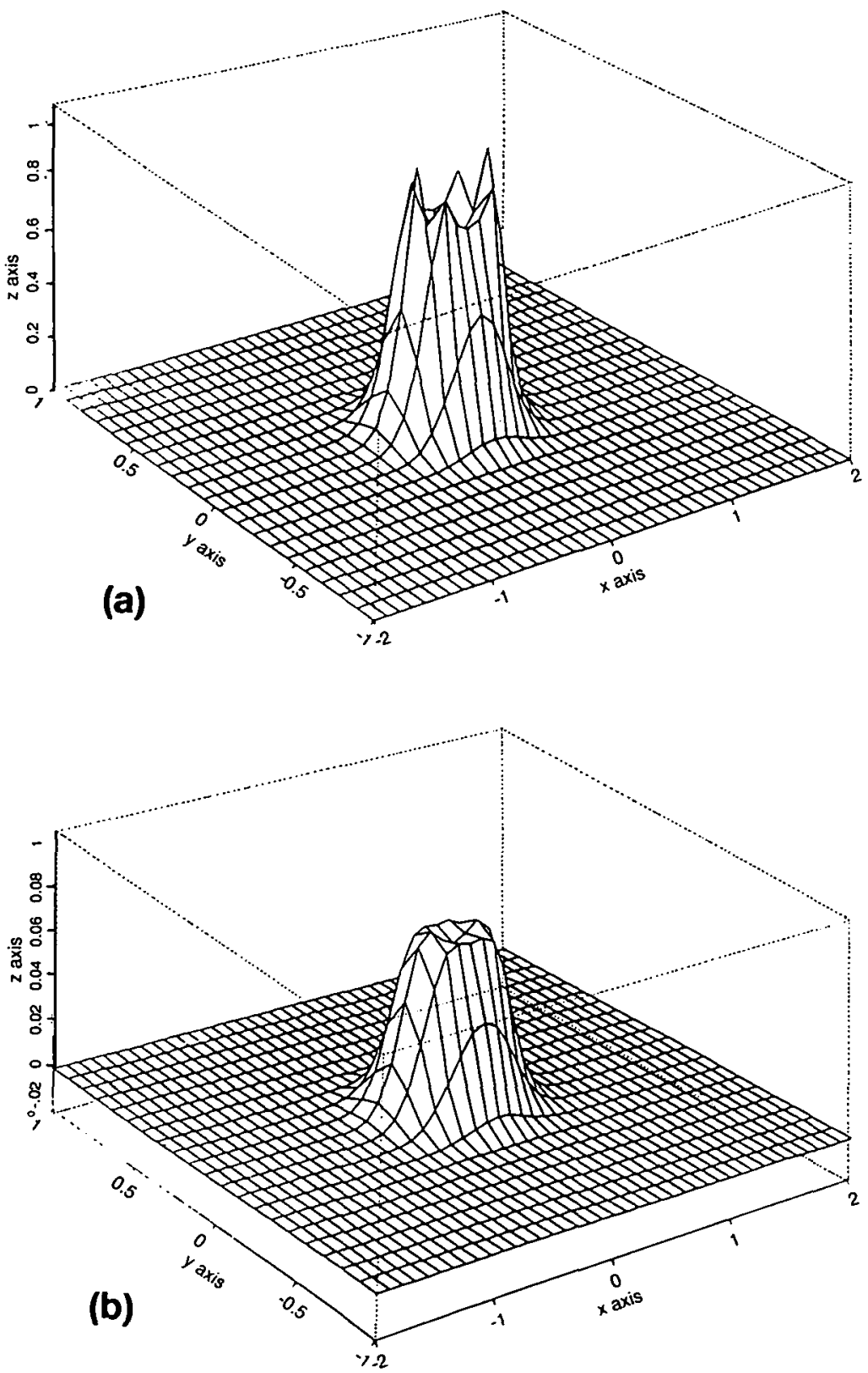

FIgURE 4. Contour plots for $u$ due to initial disturbance with a concave top in a small space. (a) $t=0$ and (b) $t=0.4$ (FEM) (for (c) and (d) see overleaf). 

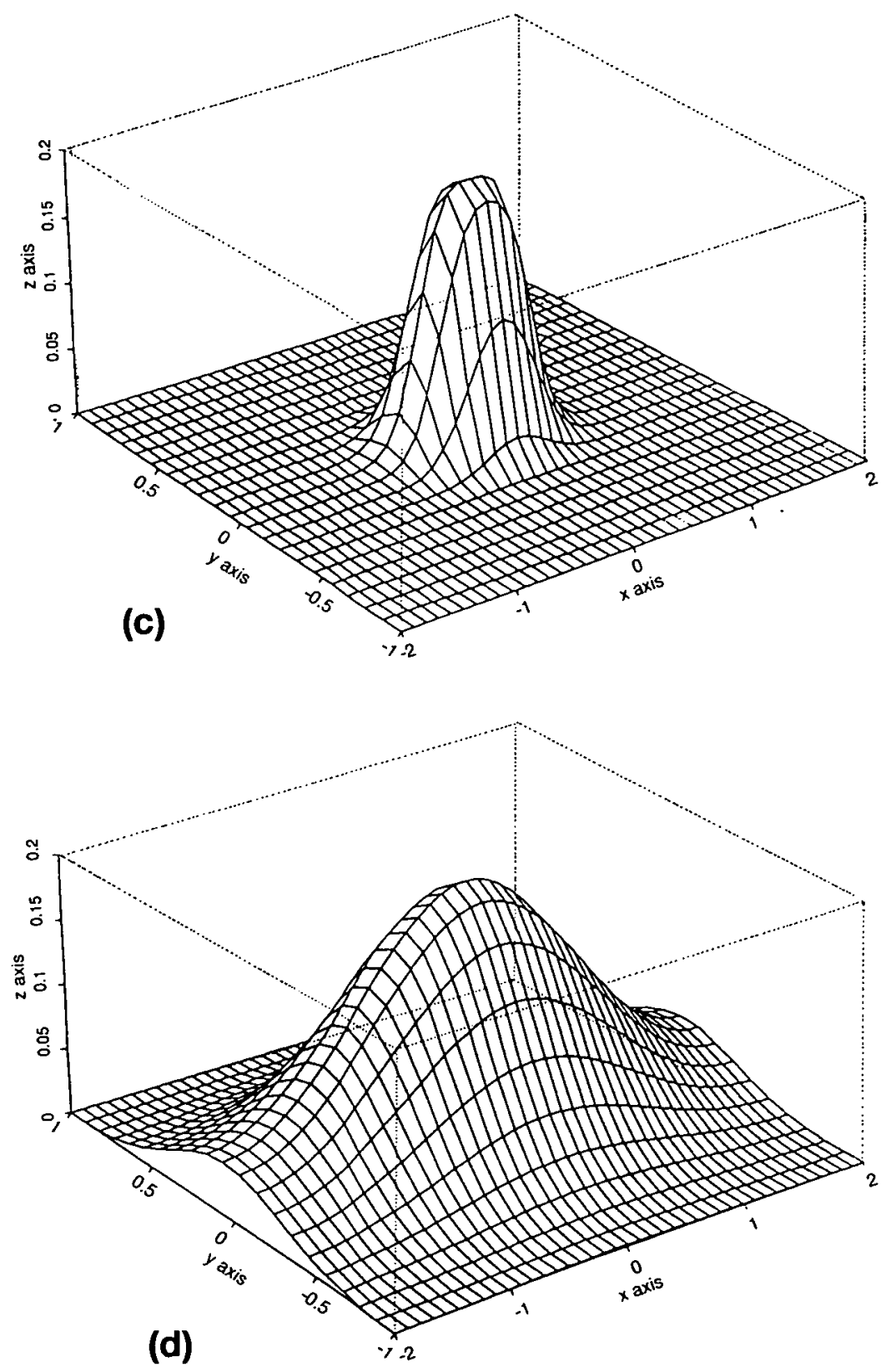

FIgURE 4. Contour plots for $u$ due to initial disturbance with a concave top in a small space. (c) $t=1$ and (d) $t=4$ (FEM) (for (a) and (b) see previous page). 
It is shown that during the spreading of the disturbance the concave part of $u$ grows and forms a hump at $t=1$. At $t=4$, we get a similar picture as in example 1 at $t=3$.

\subsection{Initial disturbance with a convex top in a small space: FDM and FEM} All parameters are the same as those in example 2, but the initial disturbance

$$
u^{0}(x, y)=\exp \left[-10\left(x^{2}+4 y^{2}\right)\right]
$$

has a convex top. Both the Galerkin FEM and simple FDM were used to solve this problem. The contour plots at $t=0,1$ and 4 are shown in Figure 5(a)(d). Figure 5(c) and Figure 5(d) are the results obtained by FDM and FEM respectively, from which we see that the schemes produce results which are in good agreement.

\subsection{Initial disturbance with convex and concave distribution and isopleths} in a large space: FDM Having seen that the FDM works well in our problem and knowing that it saves considerable CPU time, we use it to solve a problem in a large space with a more complicated initial disturbance; namely,

$$
u^{0}(x, y)= \begin{cases}x^{2}+4 y^{2}, & x^{2}+4 y^{2} \leq 1 \text { and } x \geq 0, \\ 1, & 1<x^{2}+4 y^{2} \leq 1.5 \text { and } x \geq 0, \\ \exp \left[-10\left(x^{2}+4 y^{2}-2.25\right)\right], & x^{2}+4 y^{2}>2.25 \text { and } x \geq 0, \\ 0, & x<0\end{cases}
$$

This initial disturbance has a convex part and a concave part. Noting the scaling relation in (3), and wishing to see the global behaviour of the solution in a reasonably large space, we take $\lambda_{1}=0.02, \lambda_{2}=0.01, \mu=1.0, a=10, b=8$, $h=k=0.2$ and $\tau=0.01$.

Figures 6(a)-(d) show the contour plots of $u$ at $t=0,2,10$ and 20. It is obvious that, as time goes on, the concave part of $u$ and its isopleths are going to gradually become convex, and a quasi-travelling 2-dimensional wave will result just as expected from theoretical arguments.

3.5. Long term behaviour: FDM From examples 1 to 4 we can see that various local initial disturbances will lose their initial complex structure to become a hump at the first stage and then form quasi-travelling waves at the second stage. So, to check the long term behaviour, we may choose the initial disturbance to be as simple as possible. In this example, we set $\lambda_{1}=0.02, \lambda_{2}=0.01, \mu=1.0$, $a=10, b=8, h=k=0.2, \tau=0.01$ and

$$
u^{0}(x, y)=\exp \left[-10\left(x^{2}+4 y^{2}\right)\right] \text {. }
$$




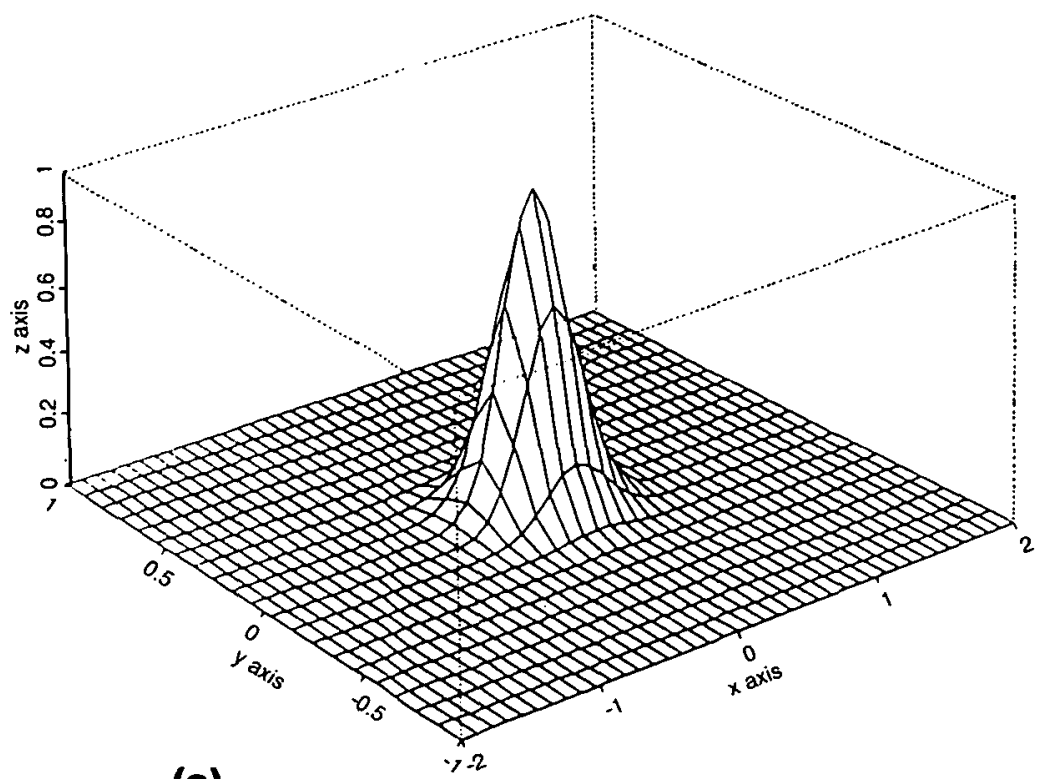

(a)

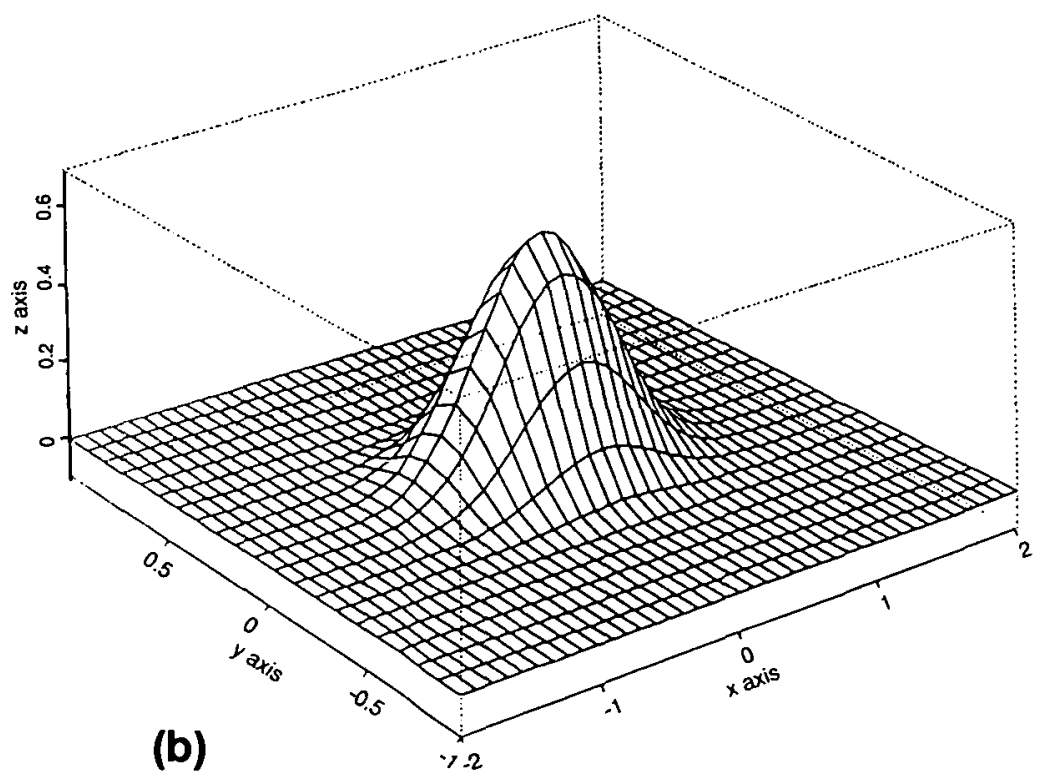

FIgURE 5. Contour plots for $u$ due to initial disturbance with convex top in a small space. (a) $t=0$ and (b) $t=1$ (FEM) (for (c) and (d) see facing page). 

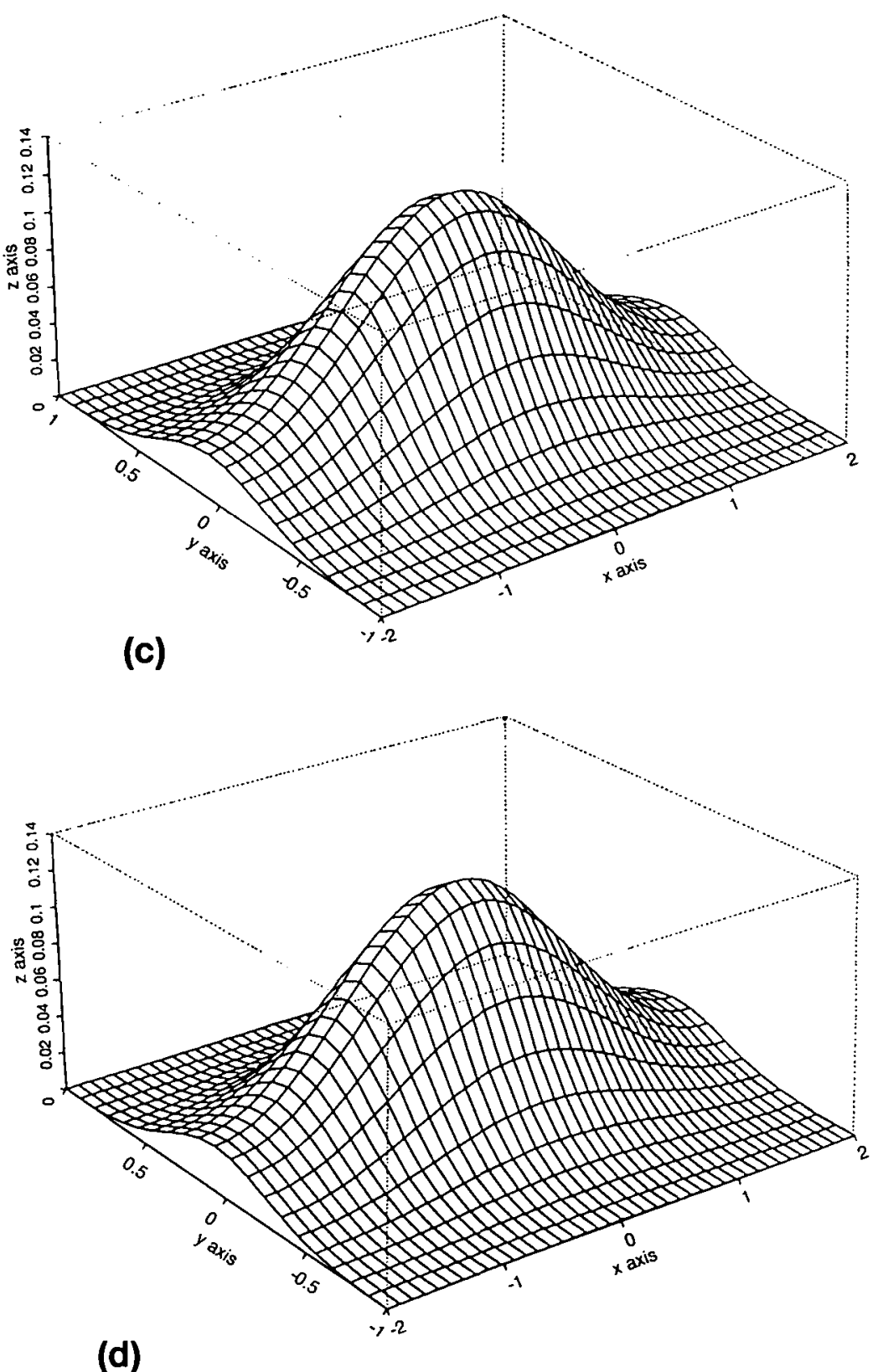

FIGURE 5. Contour plots for $u$ due to initial disturbance with convex top in a small space. (c) $t=4$ (FEM) and (d) $t=4$ (FDM) (for (a) and (b) see previous page). 

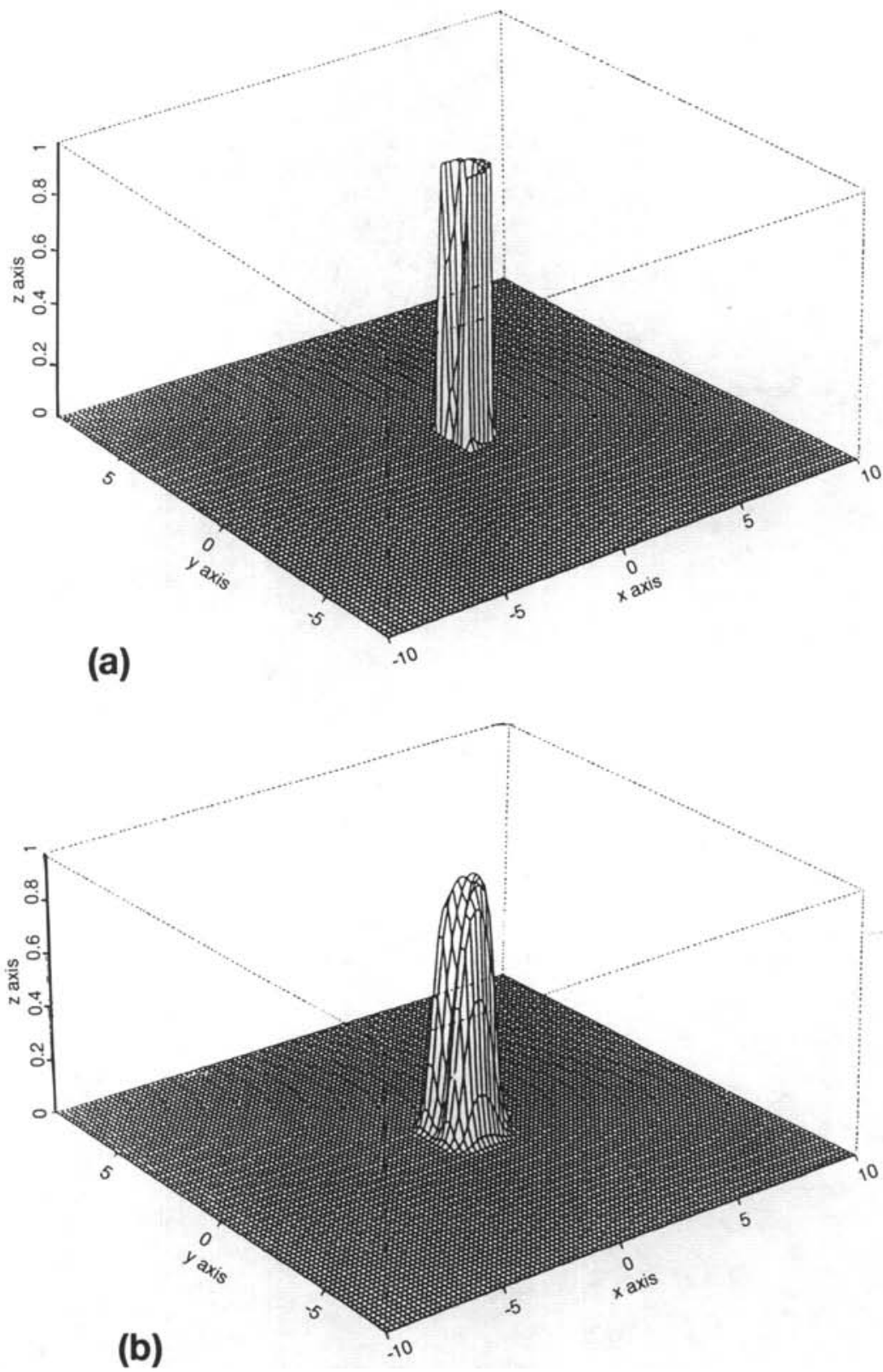

FIGURE 6. Contour plots for $u$ due to initial disturbance with convex and concave distribution and isopleths in a large space. (a) $t=0$ and (b) $t=2$ (FDM) (for (c) and (d) see facing page). 

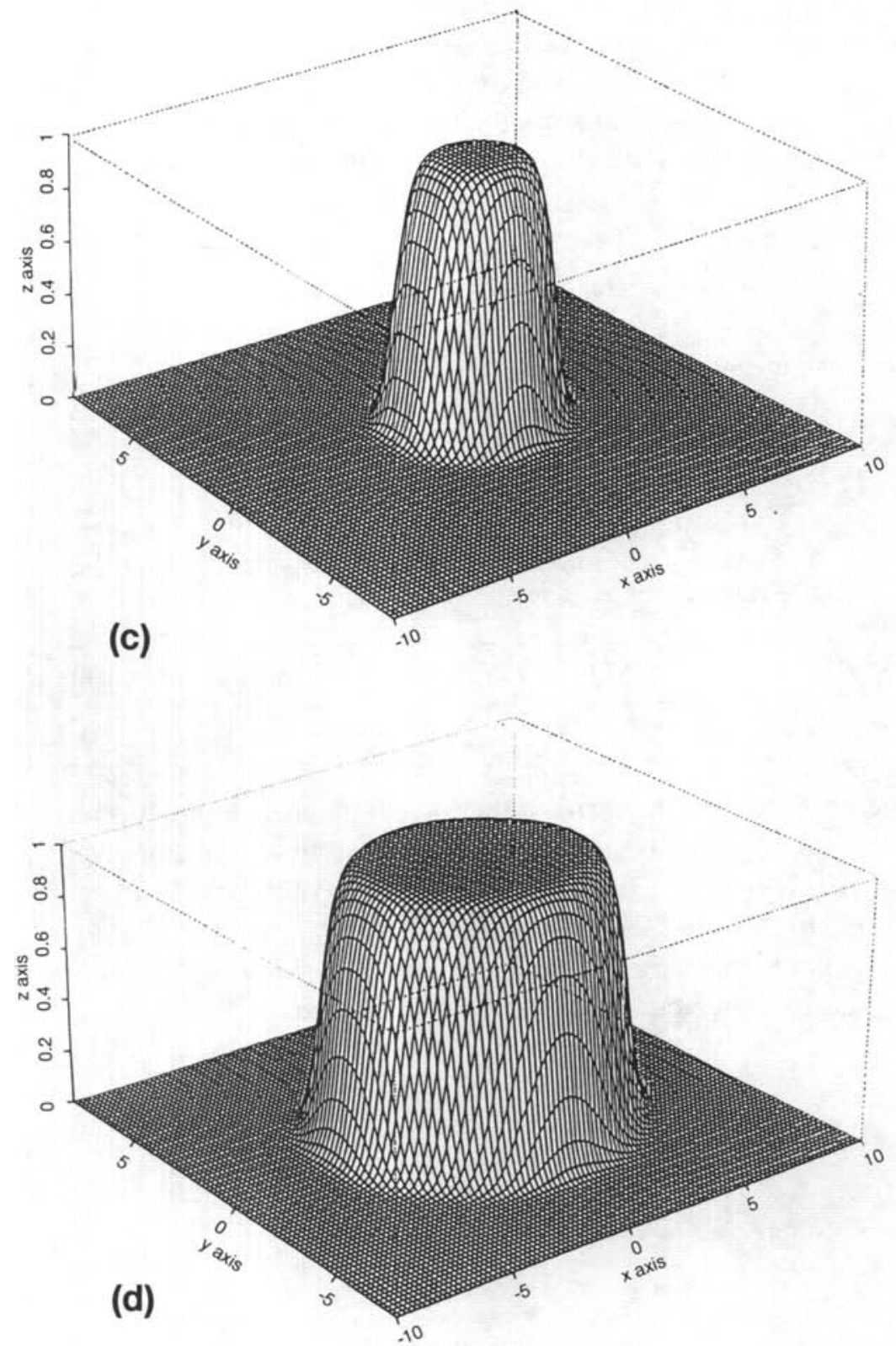

FIGURE 6. Contour plots for $u$ due to initial disturbance with convex and concave distribution and isopleths in a large space. (c) $t=10$ and (d) $t=20$ (FDM) (for (a) and (b) see previous page). 
Using the scheme from the FDM, we obtain results at $t=0,10,30,50$ as shown in Figure 7(a)-(d). We see that from $t=10$ when the quasi-wave front forms, to $t=30$, when the wave front is still far away from the boundary, we have fully developed wave front solutions, i.e., a flat hump with height 1 travelling outward. From $t=10$ to 30 , for every $t_{p}=10+p \Delta t, \Delta t=0.1$, we record $u\left(x, 0, t_{p}\right)$, and using the trapezoidal rule we calculate

$$
x_{f}\left(t_{p}\right)=\frac{1}{2} \int_{-a}^{a} u\left(x, 0, t_{p}\right) d x .
$$

This gives the mean location of the wave front at time $t_{p}$. From

$$
v\left(x_{f}\right)=\frac{x_{f}\left(t_{p}\right)-x_{f}\left(t_{p-1}\right)}{\Delta t},
$$

we get the velocity of the wave front at the position $x_{f}$. Then using our choice of $\lambda_{1}, \lambda_{2}, \mu$ and scaling according to (3) we get the velocity $V$ of the circular wave front, as a function of distance $r$, as follows.

\begin{tabular}{|c|c|c|c|c|c|c|c|c|c|c|c|}
\hline$r$ & 16.09 & 19.92 & 23.81 & 27.75 & 31.72 & 35.71 & 39.73 & 43.77 & 47.83 & 51.80 & 55.98 \\
\hline$V$ & 1.89 & 1.92 & 1.95 & 1.97 & 1.99 & 2.00 & 2.01 & 2.02 & 2.03 & 2.03 & 2.04 \\
\hline
\end{tabular}

It is clear that $V$ and the shape of the wave front change slowly with $r$. That is why we call it a circular quasi-travelling wave front. Within our range of accuracy, $V(r) \rightarrow 2$, i.e., the minimum planar wave front velocity, as proved theoretically by Aronson and Weinberger [2].

Once we get $V(r)$, using the scaling relation (3), we can calculate the velocity in the $x$ and the $y$-direction $v_{x}(x)$ and $v_{y}(y)$ for the quasi-elliptic wave front according to

$$
x=\sqrt{\frac{\lambda_{1}}{\mu}} r, \quad y=\sqrt{\frac{\lambda_{2}}{\mu}} r, \quad v_{x}(x)=\sqrt{\lambda_{1} \mu} V(r), \quad v_{y}(y)=\sqrt{\lambda_{2} \mu} V(r)
$$

which are suitable for any choice of $\lambda_{1}, \lambda_{2}$ and $\mu$.

\section{Conclusions}

Let us draw some conclusions on the 2-dimensional reaction-diffusion equation (4) and (2) with $f(u)=u(1-u)$ from what we have shown. 

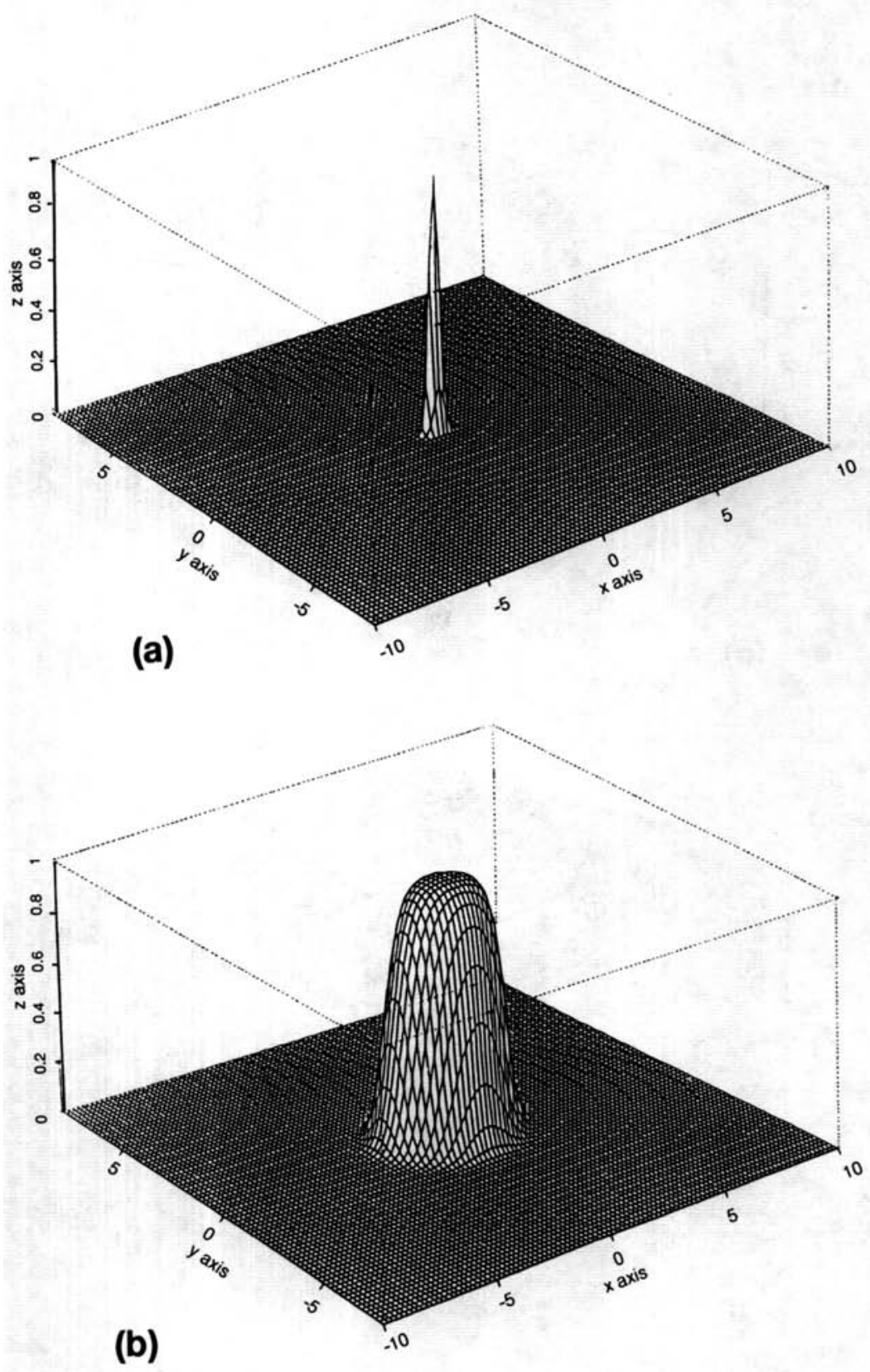

FIGURE 7. Contour plots for long term $u$. (a) $t=0$, (b) $t=10$ (FDM) (for (c) and (d) see overleaf). 

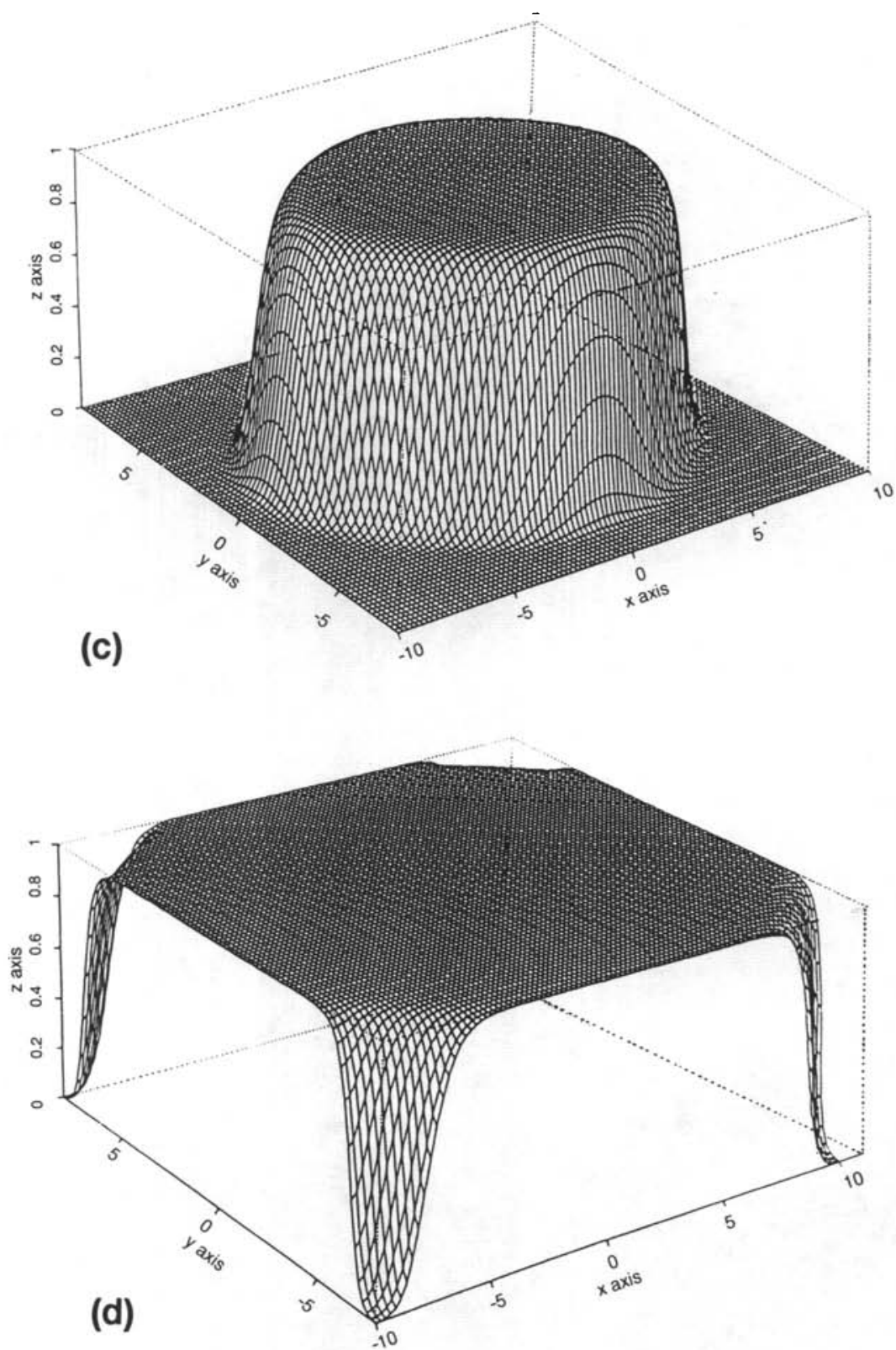

FIGURE 7. Contour plots for long term $u$. (c) $t=30$ and (d) $t=50$ (FDM) (for (a) and (b) see previous page). 
1. Any local initial disturbance will evolve according to (4) to become a hump in a larger space at the first stage, and will then form a circular quasitravelling wave front. The velocity of the front $V$ increases with distance $r$ and approaches 2, the minimum planar wave front velocity for the 1 dimensional equation. This long term behaviour is determined by the reaction and diffusion of the system itself, but does not depend on the details of the initial disturbances at all.

2. Once we get $V(r)$ and know the parameters $\lambda_{1}, \lambda_{2}$ and $\mu$ we can predict the velocity of the elliptic quasi-travelling wave front for the 2-dimensional reaction-diffusion equation (2). This could be used to model the spreading of bushfires, populations and so on.

3. All the numerical schemes shown in section II work quite well for numerical solutions to the initial-boundary value problem (6) with satisfactory accuracy and stability. The reason for this is most possibly due to the long term solution of the equation itself which has stable and organised behaviour. The FDM is very quick to use and nearly as accurate as the FEM.

\section{Acknowledgements}

One of us (S. Tang) appreciates the support of the University College of the University of New South Wales, Australia, where most of this work was completed during his visit. The advice of John Marley and Ian French on various computational issues is also appreciated. We are also grateful to V. K. Robson for initial work on the FDM.

\section{References}

[1] J. Argyris, M. Haase and J. C. Heinrich, "Finite approximation to two-dimensional sineGordon equations", Computer Methods in Appl. Mech. and Eng. 86 (1991) 1-26.

[2] D. G. Aronson and H. F. Weinberger, "Multidimensional nonlinear diffusion arising in population genetics", Advance in Mathematics 30 (1978) 33-76.

[3] R. Grimshaw and S. Tang, "The rotation-modified Kadomtsev-Petviashvili equation : an analytical and numerical study", Studies in Appl. Math. 83 (1990) 223-248.

[4] S. Tang, S. Qin and R. O. Weber, "Numerical solution of a nonlinear reaction-diffusion equation", Chinese J. of Appl. Math. and Mech. 12 (8) (1991), Chinese Edition pp.703-709; English Edition pp.751-758.

[5] S. Tang and R. O. Weber, "Numerical study of Fisher's equation by a Petrov-Galerkin finite element method", J. of Austral. Math. Soc. Series B 33 (1991) 27-38. 